\title{
Use of flexible bronchoscopy for rapid diagnosis of suspected tubercular cases in rural India
}

\author{
Sameer Singhal ${ }^{1}$, Abhay. M. Gaidhane ${ }^{2}$, Nazli Khatib ${ }^{3}$, Tripti Srivatsava ${ }^{3}$, Sanjay Diwan ${ }^{4}$, Satish \\ N. Mahajan ${ }^{4}$, Shilpa Bawankhule ${ }^{4}$, Quazi Syed Zahiruddin ${ }^{2}$
}

${ }^{1}$ Department of Chest and Tuberculosis, Datta Meghe Institute Medical Sciences, Wardha, Maharashtra, India

${ }^{2}$ Department of Community Medicine, Datta Meghe Institute Medical Sciences, Wardha, Maharashtra, India

${ }^{3}$ Department of Physiology, Datta Meghe Institute Medical Sciences, Wardha, Maharashtra, India

${ }^{4}$ Department of Medicine, Datta Meghe Institute Medical Sciences, Wardha, Maharashtra, India

\begin{abstract}
Background: Reaching a correct diagnosis is a challenge for physicians treating any of the $30 \%$ to $50 \%$ of pulmonary tuberculosis patients who have negative sputum cultures or who present with no sputum. Flexible bronchoscopy acquires special importance for these cases for whom empirical anti-tuberculosis therapy is the only option left. In our study we aimed to assess the diagnostic yield of flexible bronchoscopy in patients, suspected to have tuberculosis, whose sputum smears were negative.

Methodology: In our hospital-based cross-sectional study, 42 patients were enrolled by consecutive sampling. Flexible bronchoscopy and selective bronchial washings were done in all patients.

Results: Bronchoscopy lavage smears were positive for $M$. tuberculosis in 10 (23.8\%) patients. Fifteen (35.7\%) patients had positive culture. Conclusion: Flexible bronchoscopy has an important role in the diagnosis of patients suspected to have tuberculosis, whose sputum smears are negative or who can not produce sputum.
\end{abstract}

Key words: flexible bronchoscopy, rapid diagnosis, suspected tuberculosis

J Infect Dev Ctries 2009; 3(11):860-864.

(Received 26 February 2009 - Accepted 23 October 2009)

Copyright $(92009$ Singhal et al. This is an open-access article distributed under the Creative Commons Attribution License, which permits unrestricted use, distribution, and reproduction in any medium, provided the original work is properly cited.

\section{Introduction}

Tuberculosis (TB) causes approximately two million deaths per year, $98 \%$ of which occur in lowincome countries [1]. India has the highest TB burden of any country in the world [2,3]. The widespread implementation of the internationally recommended Directly Observed Treatment, Short-course (DOTS) strategy has proved to be an effective tool in controlling TB on a mass basis and is being practiced in over 180 countries [4-6].

Sputum microscopy continues to be the best tool for detection of infectious TB [7] in patients with a compatible clinical picture; however, sputum smears do not reveal acid-fast bacilli (AFB) in all patients, and a "smear-negative/culture-positive" state has been observed in $22 \%$ to $61 \%$ of the cases [8-10]. The DOTS approach has provided access to standardised, quality assured microscopy [11].
However, sputum smear-negative pulmonary tuberculosis (SSN-PTB) still remains a common problem. This is particularly true in immunosuppressed patients, such as those with acquired immunodeficiency syndrome (AIDS) in whom SSN-PTB is quite common. Various methods have been employed to ascertain active TB disease in patients with suspected SSN-PTB, including mathematical modelling for predicting active disease $[12,13]$. Of these, bronchoscopic procedures have been studied in some detail.

Flexible bronchoscopy has proven to be a useful technique in the evaluation of suspected cases of pulmonary tuberculosis that are sputum smearnegative $[14,15]$. The present study tested the sensitivity of bronchial wash for rapid diagnosis of tuberculosis in sputum smear-negative suspected tubercular patients. The study further aimed to assess the diagnostic yield of flexible bronchoscopy in 
patients, suspected to have tuberculosis, whose sputum smears were negative.

\section{Materials and methods}

Study Settings

This study was conducted at a 909-bed tertiary care teaching hospital, Wardha of Datta Meghe Institute of Medical Sciences University (National Assessment of Accreditation Council Accredited Grade A). The hospital provides health care to the nearby area, mainly from the rural area of Wardha district and other parts of Central India. This hospital has a DOTS center and designated microscopy center which implements quality assurance protocol and provides quality assured laboratory services. An built-in routine system has been designed for sputum microscopy External Quality Assessment (EQA) and for the supervision and monitoring of the diagnostic systems from the Revised National TB Control Programme (RNTCP) by the chest and tuberculosis specialist in the Department of Chest Disease. The program is run per the guidelines of RNTCP. Sputum microscopy is examined by a trained technician under the supervision of the senior Tuberculosis Laboratory supervisor.

\section{Study Design}

This study is a hospital-based, cross-sectional study.

\section{Study period of recruitment}

The study period of recruitment was between 1 February 2007 and 30 March 2008.

\section{Ethical Issues}

The study protocol was approved by the institutional ethical committee.

\section{Method of Selection}

Recruitment was done by using consecutive sampling in which the patients were chosen on a strict "first come, first chosen" basis to the Department of Chest Disease. All individuals who were eligible were included as they were seen.

\section{Eligibility Criteria}

The eligible individuals were the adult patients suspected of having pulmonary tuberculosis who presented negative sputum smears (in at least three samples), and who were registered under RNTCP (already taking DOTS). The suspected tuberculosis patients were the individuals in whom sputum smears were smear-negative and who showed symptoms of cough for more than three weeks, evening rise of temperature, and loss of weight. The diagnosis of sputum smear-negative pulmonary tuberculosis was based on the following criteria: at least three negative sputum smears (including at least one early-morning specimen); chest radiography findings consistent with tuberculosis; and lack of response to a trial of broadspectrum antimicrobial agents. Because the fluoroquinolones are active against $M$. tuberculosis and thus may cause transient improvement in persons with tuberculosis, they were avoided. In persons with known or suspected HIV infection, the diagnostic evaluation was expedited. Patients younger than 18 years were excluded from the study.

\section{Data Collection}

In 13 months, 144 patients in the DOTS centre were diagnosed with smear-negative pulmonary tuberculosis. Every third patient was eligible for inclusion in the study; therefore, a total of 48 patients were eligible but only 42 participants gave consent and were subsequently included in the study. The response rate was $88 \%$.

The purpose of the study was explained to the patients and those who consented to participate were included. Others, who left the interview and examination prematurely for various reasons, were excluded from the study. Rapport was developed with the participants and data was collected using a structured interview schedule and examination by a trained and experienced chest physician. Patients suspected of having pulmonary tuberculosis and presenting negative sputum smears (in at least 3 samples) were evaluated using flexible bronchoscopy. Participants were evaluated according to a pre-designed protocol. The clinical profile and radiological lesions in these patients were consistent with the diagnosis of pulmonary tuberculosis but three consecutive occasions produced specimens of sputum that were negative for AFB.

Forty-two patients who were suspected to have pulmonary tuberculosis with sputum smear-negative were enrolled in the study. Flexible bronchoscopy and selective bronchial washings were performed on all patients. Flexible bronchoscopy was conducted trans-nasally.

Prior to the procedure, the patients were given pre-medication with sedatives, opiates and atropine. 
Not more than $30 \mathrm{ml}$ of $4 \%$ topical Xylocaine and no vasoconstrictors were administered during the exam. After inspection of the airways, the bronchoscope was wedged in areas of maximal involvement, as evident on chest roentgenogram. The bronchoalveolar lavage (BAL) consisted of five or six $\mathrm{ml}$ aliquots of normal saline solution; fluid was aspirated using mechanical suction.

Bronchial washings were obtained from the affected parts. Mucosal biopsies were done in patients in where endobronchial abnormalities were noted. Transbronchial biopsies were performed in selected patients from the radiological localizations. Ziehl-Nielsen staining and culture in LowensteinJensen medium were the microbiological studies. Aliquots of BAL fluid were sent for staining of the cell pellet in the cytology laboratory.

To prevent cross-contamination, guidelines such as those issued by the British Thoracic Society regarding bronchoscopy and infection control [16] and the American College of Chest Physicians and the American Association of Bronchology [17] were followed.

\section{Results}

Among the 42 patients, 28 (66.7\%) were males. The average age was $38.4 \pm 15.3$ years. Table 1 shows that only 15 patients $(35.7 \%)$ out of 42 patients had tuberculosis. Twenty-seven (64.2\%) patients were confirmed with diagnoses other than tuberculosis. The diagnoses were community-acquired pneumonia (CAP) in 18 patients (42.9\%), foreign body in five (11.9\%), bronchial carcinoma in three (71.4\%) allergic bronchopulmonary aspergillosis in one patient $(2.4 \%)$.

Table 1. Bronchoscopy involved in diagnosis

\begin{tabular}{|l|c|}
\hline Diagnosis & No (\%) \\
\hline Pulmonary Tuberculosis & $15(35.7)$ \\
\hline Community Acquired Pneumonia & $18(42.9)$ \\
\hline Foreign Body & $5(11.9)$ \\
\hline Bronchial Carcinoma & $3(7.2)$ \\
\hline Allergic bronchopulmonary aspergillosis & $1(2.3)$ \\
\hline Total & $\mathbf{4 2}(\mathbf{1 0 0 . 0})$ \\
\hline
\end{tabular}

\section{Discussion}

When there is suspicion of active tuberculosis, patients in whom direct examination of sputum is inconclusive represent a diagnostic and therapeutic challenge. Our study confirms this observation since other infectious, neoplastic and foreign bodies (beetel nut which was extracted from all the patients; retrospectively, all patients gave history of beetel nut chewing while going to sleep) were diagnosed in these patients $(64 \%)$ who were originally thought to be suffering from pulmonary tuberculosis.

Rao [18] has demonstrated the usefulness of bronchoscopy among smear-negative patients in India. Of 55 sputum smear-negative patients, 15 $(27.3 \%)$ had AFB-positive bronchial washings (cultures were not performed), and bronchial carcinoma was diagnosed in another five patients. In our study it was found that bronchoscopic lavage smears were positive for M. tuberculosis in 10 (24\%) patients and 15 (36\%) patients had positive culture. Similar results were seen in a study conducted by Yuksekol et al. [19], in which Bronchoscopic lavage smears were positive for M. tuberculosis in 13 (23\%) patients and $28(50 \%)$ patients had positive culture. Using bronchoscopic procedures early in the diagnosis was performed in $27(48.2 \%)$ patients. Bronchoscopic procedures provided overall diagnostic yields in $62.7 \%$ (23/43) of the patients. A study conducted by Charoenratanakul et al. [20] shows that bronchoscopic procedures provided overall diagnostic yields in $47.5 \%$ (19/40) of the patients. The diagnostic yield of overall bronchoscopic procedures for tuberculosis in this study was $35.7 \%(15 / 42)$ of patients. It consisted of positive BAL smear in $23.8 \%(10 / 42)$ of patients and positive for mycobacterial culture in $35.7 \%(15 / 42)$ of patients. Non-tuberculosis conditions were diagnosed by the bronchoscopic method in 27 patients (64.2\%).

In the present study, bronchoscopy was useful not only for the diagnosis of tuberculosis but also for the identification of other pathologies, especially neoplasia (3 cases) and foreign bodies (5 cases), whose delayed diagnosis may exclude the possibility of a surgical cure. Some authors whose objectives were similar to those of our study have also reported diagnosing neolpalsia after performing bronchoscopy [21]. Other infectious diseases such as CAP and 
aspergillosis were also diagnosed through bronchoscopy during this study.

The limitation in this study was that no investigative method other than bronchoscopy was used for detection of tuberculosis. Therefore, no comparative study was possible. The sensitivity of bronchoscopy for tuberculosis and the determination of the diagnosis of other pathologies (neoplasia, infectious diseases or diseases mediated by the hypersensitivity mechanism) reinforce data in the literature indicating this type of examination when there is suspicion of tuberculosis but no confirmation in the sputum microscopy. This study though cannot be generalized as it was conducted in a tertiary care hospital setup.

High prevalence settings for TB are often resource-limited and thus offer constraints for the use of bronchoscopy. There would be a need for further research on the feasibility and cost-effectiveness of bronchoscopy for TB diagnosis in resource-limited settings before it can be recommended as a useful tool. Involving medical colleges as partners and implementing Public Private Partnership are ways in which big hospitals can cater to the needs of the patients. In such setups, flexible bronchoscopy through the RNTCP can be one approach to prevent needless governmental expenditures on TB diagnosis and loss of disability-adjusted life years (DALY) in patients.

\section{Conclusion}

Although spread of TB following bronchoscopy has occasionally been reported, flexible bronchoscopy has an important role in the diagnosis of patients suspected to have tuberculosis, whose sputum smears are negative or who can not produce sputum. This study suggests that in tertiary care hospitals of areas with high TB prevalence, bronchoscopic procedures should be performed in those cases in which other diagnoses such as malignancy, foreign body, etc. must be ruled out.

\section{References}

1. Maher D, Raviglione M (2005) Global epidemiology of tuberculosis. Clin Chest Med, 26: 167-82.

2. Planning and Financing (2007). Global Tuberculosis Control;Surveillance. WHO Geneva: WHO Report.

3. TB: Burden of the disease in India (2008). RNTCP Status Report TB India. Central TB Division, Directorate General of Health Services 10. http://www.tbcindia.org.
4. WHO Stop TB Strategy (2008). RNTCP Status Report TB India. Central TB Division, Directorate General of Health Services $14 \mathrm{p}$.

5. Treatment of Tuberculosis: Guidelines for National Programmes (2003). World Health Organization, Stop TB Department. 3rd edn. World Health Organization Geneva:; WHO/CDS/TB/2003.313 p.

6. TB India. (2007). RNTCP Status Report. New Delhi: Central TB Division, Directorate General of Health Services, Ministry of Health and Family Welfare, Government of India

7. TB India. (2008). WHO Stop TB Strategy. RNTCP Status Report, March. Central TB Division, Directorate General of Health Services 14 p.

8. Hong Kong Chest Service/Tuberculosis Research Center Madras/British Medical Research Council (1979) Sputum smear negative tuberculosis: controlled clinical trial of 3month and 2-month regimen of chemotherapy (first report). Lancet 1: 1361-3.

9. Narain R, Subbarao MS, Chandrasekhar P, Pyarelal (1971) Microscopy positive and microscopy negative cases of pulmonary tuberculosis. Am Rev Respir Dis 103: 761-3.

10. Kim TC, Blackman RS, Heatwole KM, Rochester DF (1984) Acid fast bacilli in sputum smears of patients with pulmonary tuberculosis: prevalence and significance of negative smears pretreatment and positive smears post treatment. Am Rev Respir Dis 29: 264-8.

11. Sharma SK, Liu JJ (2006) Progress of DOTS in global tuberculosis control. Lancet 367: 951-2.

12. Mello FC, Bastos LG, Soares SL, Rezende VM, Conde MB, Chaisson RE, et al. (2006) Predicting smear negative pulmonary tuberculosis with classification trees and logistic regression: a cross-sectional study. BMC Public Health 6: 43.

13. Tattevin P, Casalino E, Fleury L, Egmann G, Ruel M, Bouvet E (1999) The validity of medical history, classic symptoms, and chest radiographs in predicting pulmonary tuberculosis: derivation of a pulmonary tuberculosis prediction model. Chest 115: 1248-53.

14. 1 Danek SJ, Bower JS (1979) Diagnosis of pulmonary tuberculosis by flexible Flexibleoptic bronchoscopy. Am Rev Respir Dis 119: 677-79.

15. Wallace J, Deutsch A, Harrell J, Moser K (1981) Bronchoscopy and transbronchial biopsy in evaluation of patients with suspected active tuberculosis. Am J Med 70:1189-94.

16. Woodcock A, Campbell I, Collins JVC (1989) Bronchoscopy and infection control. Lancet 2: 270-1.

17. Mehta AC, Prakash UB, Garland R, Haponik E, Moses L, Schaffner W et al. (2005) American College of Chest Physicians and American Association for Bronchology [corrected] consensus statement: prevention of flexible bronchoscopy-associated infection. Chest 128: 1742-55.

18. Rao S (1993) Significance of bronchial washings smears negativity in 'suspect' pulmonary tuberculosis. Trop Doct 23: $170-171$.

19. Yüksekol I, Bal S, Ozkan M, Balkan A, Bedirhan I, Tozkoparan E, Demirci N, Seber O (2003) The value of Flexibleoptic bronchoscopy in diagnosis of smear negative pulmonary tuberculosis. Tuberk Toraks. 51: 405-9.

20. Charoenratanakul S, Dejsomritrutai W, Chaiprasert A (1995) Diagnostic role of Flexibleoptic bronchoscopy in suspected 
smear negative pulmonary tuberculosis. Respir Med 89: 621-3.

21. Caymmi A, S ilveira M, Montal G, Lemos A. (2004) Role of the Flexibleoptic bronchoscopy in the diagnosis of patients with suspected pulmonary. J Bras Pneumol [online] 30: 1, 39-45. www.scielo.br/pdf/jbpneu/v30n1/en_v30n1a08.pdf

\section{Corresponding Author}

Dr. Quazi Syed Zahiruddin

Datta Meghe Institute Medical Sciences

Wardha, Maharashtra, India

91-9765404065 (Mobile)

91-7152-287713

Email: zahirquazi@ rediffmail.com

Conflict of Interest: No conflict of interest is declared. 